\title{
An Auction-Based Dispatching Method for an Electronic Brokerage of Truckload Vehicles
}

\author{
Jun Ho Lee, Kap Hwan Kim* \\ Department of Industrial Engineering, Pusan National University, Busan, Korea
}

(Received: November 24, 2014 / Revised: February 12, 2015 / Accepted: February 16, 2015)

\begin{abstract}
This study suggests an electronic brokerage system that has the capability of efficiently matching dispatching delivery tasks with trucks. In the brokerage system, individual truck drivers and shippers are allowed to participate in the dispatching process through the internet or wireless communication. An auction-based dispatching method for the electronic brokerage system is suggested. The basic rationale of the auction-based dispatching method-which is a distributed decision-making process-is discussed. The performance of the suggested algorithms is evaluated by a simulation study.
\end{abstract}

Keywords: Auctions, Truck dispatching, Assignment problem, Brokerage

* Corresponding Author, E-mail: kapkim@pusan.ac.kr

\section{INTRODUCTION}

Because the average size of Korean trucking companies is relatively small, most individual truckers depend heavily on brokerage companies for obtaining delivery tasks and usually pay high brokerage fees to brokerage companies. More than $90 \%$ of Korean trucking companies operate privately-owned trucks, and truckers obtain more than $65.6 \%$ of delivery tasks through brokers (Ha and Kim, 2003). Recently, many electronic brokerage systems in Korea have opened for the purpose of providing various services that include searching for idle trucks and freights, monitoring the status of freights and trucks, dispatching trucks, reporting delivery completions, and reporting the tariffs.

The efficiency of a brokerage system depends highly on the dispatching function, which matches a delivery request with an available truck. In spite of the importance of the dispatching function, little research has been done to develop an algorithm for the brokerage systems. Although there have been several published papers, most of them assume that trucks are associated with a company in which individual constraints or conditions that each truck driver has are usually ignored.
To investigate the feasibility of electronic marketing to the brokerage of the transportation business, Beilock and Shell (1992) discussed the results of a survey. They found that electronic marketing of transportation services is technically feasible and would be welcomed by many in the industry. It was also found that this technology has the potential for greatly lowering transaction costs, thereby reducing the overall costs of marketing and improving the efficiency of the transportation cost. It was also found that the electronic marketing systems might promote direct negotiations between shipper/receivers and carriers.

Much research about the truck-dispatching problem has been conducted during the last several decades. However, only previous studies that are directly related to "dynamic dispatching" will be introduced. Psaraftis (1988, 1995) explained the unique properties of "dynamic" vehicle-routing problems, which are different from those of static vehicle-routing problems, and illustrated various examples of real problems of the dynamic problems. It should be noted that the dispatching problem in this study can be considered as a vehicle-routing problem in a broad sense and deals with a dynamic situation. An algorithm was also suggested for the dynamic routing of 
cargo ships.

In studies by Frantzeskakis and Powell (1990), Powell and Carvalho (1995), Powell (1996), and Carvalho and Powell (2000), a formulation was suggested for optimally allocating vehicles to delivery tasks. The formulation was termed as a logistics queueing network and used a linear approximation of the future values of vehicles.

In studies by Gendreau et al. (1995, 1996a, 1996b) and Laporte and Louveaux (1993), stochastic vehiclerouting problems were addressed in which some elements of the problem are random. Common examples of the random elements are stochastic demands and stochastic travel times. Although the stochastic vehiclerouting problem can be considered as either static or dynamic, the solution technique can be easily applied to dynamic situations.

The truckload vehicle dispatching problem, which is related to the dispatching algorithm in this paper, has been intensively studied with the name of pickup and delivery problems. Some examples are studies by Wang and Regan (2002), Gronalt et al. (2003), Gendreau et al. (2006), Gribkovskaia et al. (2007), Imai et al. (2007), and Parragh et al. (2008a, 2008b).

Powell and Gittoes (1996) developed a dynamic task-assignment algorithm for truck-load transportation. Shen et al. (1995) applied the neural network model to the dispatching of LTL trucks. In their study, a neural network was constructed based on historic decisions of human dispatchers. Madsen et al. (1995) suggested a heuristic algorithm for a dial-a-ride problem, which is one example of a dynamic vehicle-routing problem. Ichoua et al. (2000) addressed a real-time vehicle-dispatching problem with a diversion possibility in which diverting a vehicle away from its current destination in response to a new customer request is allowed.

Fischer et al. (1996) suggested a multi-agent approach for solving transportation problems. They showed how the auction mechanism can be applied to solve the vehicle-routing problem and dynamically revise the initial solution of the vehicle-routing problem by considering continuously changing situations. Kutanoglu and $\mathrm{Wu}$ (1999) also argued that an auction algorithm can be used for scheduling manufacturing resources and suggested a pricing mechanism based on the Lagrangean multipliers. Bertsekas (1981, 1988, 1990) discussed how the assignnment problem can be solved in a distributed manner by using the auction algorithm.

The contribution of this paper is to apply the auction algorithm to the brokerage system for dispatching full load trucks. Also, this paper proposed a method to apply the auction algorithm even when deliver tasks and trucks arrive at the system dynamically, which is another contribution of this paper.

The next section suggested a brokerage system with a distributed dispatching capability. Section 3 introduces an auction-based technique for solving the assignment problem. This section also suggests an auction-based te- chnique for solving the assignment problem as a method for dispatching trucks. Section 4 discusses the performance of the auction-based dispatching procedure. Conclusions are provided in the final section.

\section{A BROKERAGE SYSTEM WITH A DISTRIBUTED DISPATCHING CAPABILITY}

In this study, the brokerage system is assumed to include an information center, shippers (or receivers), and trucks. The study excludes receivers of freights from the brokerage system and assumes that shippers represent both shippers and receivers. In principle, dispatching decisions are made by exchanging bids among shippers and trucks through the brokerage system. The minimal functions that the information center must have are transmitting, receiving, and storing data on trucks and delivery tasks. That is, having the decision-making function is not a requirement for an information center. However, when trucks or shippers are permitted to consign their transactions to the brokerage system, part of the algorithm for bid construction can be imbedded into the software of the brokerage system.

The procedure in which trucks are matched with delivery tasks is as follows. A shipper sends a delivery application to the information center. The application information includes the place of pickup, the destination, a time window for pickup or delivery, and other conditions. The information center monitors and maintains information about delivery tasks and trucks, and provides this information to shippers and trucks.

Trucks must be equipped with mobile computer terminals. Trucks or shippers obtain data from the information center about posted tasks or trucks that are involved in the dispatching process. The data about a posted task include the current lowest-bid for the task, while the data about a truck include the current highest margin that the corresponding truck can earn by performing one of the candidate tasks. Figure 1 summarizes the overall configuration of the brokerage system.

The dispatching process in this paper is dynamic in the sense that the dispatching process is triggered whenever the driver of a truck requests a delivery task or the shipper of a new freight requests a truck. Refer to Figure 2. Suppose that a truck driver (truck 4) wants a delivery task to be assigned. The truck driver selects a delivery task (task c), from the list of posted delivery tasks, for which the highest margin is expected. The truck driver (truck 4) informs the information center of its decision. Then, the information center transmits the new decision to the shipper of the selected delivery task (task c). If the shipper agrees with the new assignment, then he/she sends a cancellation notice to the driver of a previously assigned truck (truck 3 ) through the information center so that the driver of truck 3 can start looking for another delivery task, and so on. This procedure is repeated until 
no more than one delivery task is assigned to a truck and no more than one truck is assigned to a delivery task. When a shipper of a new freight requests a truck, similar things happen.

Although the dispatching decisions change whenever a truck driver or a new delivery task requests a new assignment, a deadline for committing a decision for a truck or a delivery task may exist. At the deadline for a commitment, the current assignment of a truck (task) to a task (truck) is fixed or the delivery task whose deadline for the pickup is over is removed from the list of candidate tasks.

The advantages of the brokerage system with a distributed dispatching capability may be summarized as follows: firstly, because each trucker is an independent profit maker, it should be allowed for him/her to be involved in making a decision for selecting delivery tasks, which is possible in the proposed dispatching system. Note that, in the proposed system, each trucker attempts to maximize his/her own profit in selecting delivery tasks and so he/she is responsible in his/her assignment to delivery tasks, while a centralized dispatching system assumes that truckers have to follow the dispatching decisions from the central system, which may not be reasonable for competing profit makers. Secondly, in a centralized dispatching system, the information center deals with individual truckers, continuously changing conditions or constraints of individual trucks, which may affect the dispatching decisions, may not be known to the information center. However, in the auction-based dispatching process proposed in this paper, because each truck driver and each shipper directly participates in the decision process of this study, these local conditions or constraints may be considered during the dispatching process. For example, the shipper of delivery task "c" in Figure 2 can reject the bid from truck " 4 " if loading and unloading operation of truck 4 cannot be performed at the pickup location of delivery task c because of a sudden breakdown of the corresponding handling equipment, which is not known to the information center. Thirdly, a new delivery task arrives or a new truck enters into the system, which will happen frequently, the assignment problem for all the delivery tasks and the available trucks does not have to be solved, which may take a long computational time. In the proposed approach, only a limited number of matches between trucks and delivery tasks may be changed, which requires a much shorter processing time.

The current practice of dispatching trucks in brokerage companies is as follows: when a truck driver requests a delivery task, the dispatcher reviews the list of available delivery tasks and selects an appropriate delivery task. When a shipper requests a truck, the dispatcher selects a truck among waiting idle trucks. Thus, it is attempted for a delivery task to be matched with one of multiple trucks or for a truck with one of multiple delivery tasks. However, in this study, the truck-dispatching problem is treated a problem of assigning multiple delivery tasks to multiple trucks. Future idle trucks, which are currently performing delivery tasks, as well as currently idle trucks, are considered in the dispatching decision. Thus, a more efficient assignment from the perspective of the cost is expected.

When a decision is made about the assignment of hundreds of trucks to hundreds of delivery tasks, it is time-consuming to solve an optimization problem whenever a new delivery task arrives at the shop or a truck becomes a candidate for the dispatching. The distributed dispatching process in this study localizes the changes in the dispatching decisions so that the assignment problem for all the tasks and trucks need not be solved repeatedly. Refer to Figure 2. The assignments for delivery tasks, a and $b$, remain unchanged by the arrival of a new truck 4 . For an arrival of a truck at a terminal in a city, it would

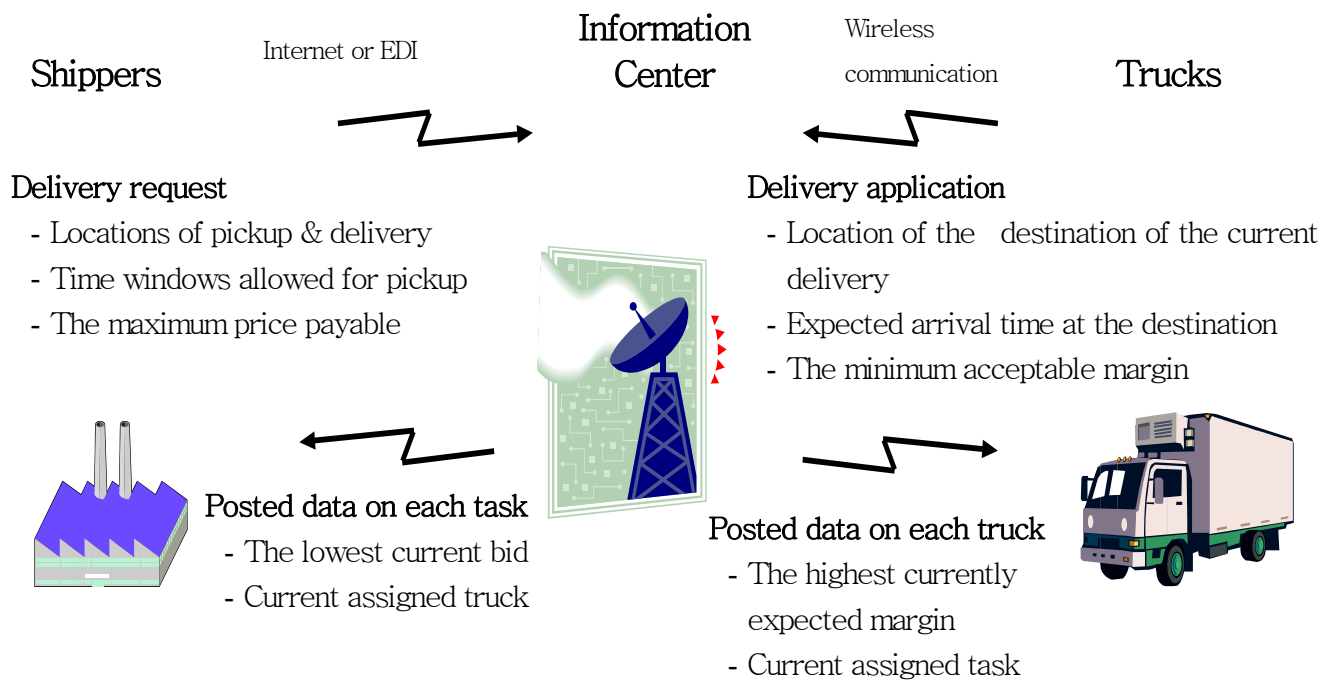

Figure 1. An electronic brokerage system considered in this study. 

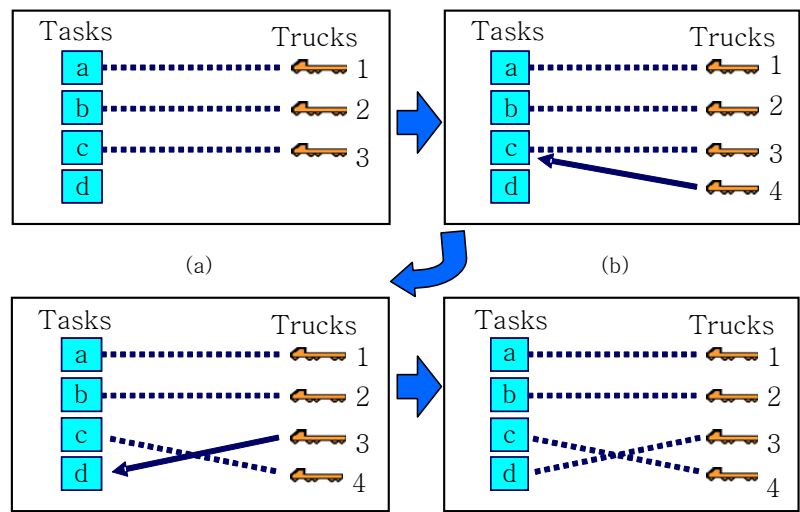

(c)

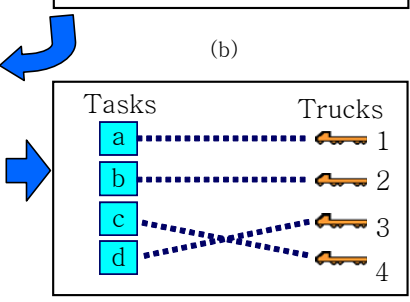

(d)
Figure 2. Dispatching process triggered by a new truck.

not be necessary to reconsider the assignment of trucks in most of the other cities that are located in long distances. Also, a truck can enter into or leave from the brokerage system without disturbing the assignment or the dispatching procedure. And note that no central controller needs to actively participate in the auction process.

\section{APPLYING AN AUCTION-BASED ASSIGNMENT ALGORITHM TO DISPATCHING PROBLEM}

This section presents an attempt to solve the dispatching problem through a market mechanism (Bertsekas, 1990), viewing each task manager (a software) or truck manager as an economic agent acting for its own best interest. This study considers the dispatching problem as an assignment problem in which multiple delivery tasks are matched with multiple trucks and the objective function is minimizing the total transportation cost. The following notations will be used to describe the auctionbased dispatching algorithm:

$\mathrm{n}$ : The number of candidate trucks for the dispatching

$\mathrm{m}$ : The number of candidate delivery tasks for the dispatching

$\mathrm{a}_{\mathrm{ij}} \quad$ : The cost of assigning truck $\mathrm{i}$ to task $\mathrm{j}$. When the objective function is the total empty travel time, this is the empty travel time for truck i to travel from the immediate destination to the pickup location of delivery task $\mathrm{j}$. When the objective function is the total response time, this is the response time for truck $\mathrm{i}$ from the moment when it is assigned to task $\mathrm{j}$ to the moment when it arrives at the pickup position of task $\mathrm{j}$.

$\mathrm{x}_{\mathrm{ij}} \quad: 1$, if truck $\mathrm{i}$ is assigned to delivery task $\mathrm{j} ; 0$, otherwise

$p_{j}^{0}$ : The maximum price that the shipper of delivery task $\mathrm{j}$ can pay. To obtain $\mathrm{p}_{\mathrm{j}}^{0}$, the loaded travel cost for task $\mathrm{j}$ is subtracted from the original maximum price

A(i) : The set of tasks to which truck i can be assigned. This set of tasks is defined by considering various local constraints that truck $i$ has in selecting tasks. Those constraints are local in the sense that they may not be known to the other trucks, shippers, or the brokerage system.

$\mathrm{V}(\mathrm{j})$ : The set of trucks to which task $\mathrm{j}$ can be assigned. This set is also defined by the local constraints of task j

When the number of trucks and the number of delivery tasks are not the same, dummy trucks or tasks will be added, and the cost coefficients $\left(a_{i j}\right)$, which are related to the dummy trucks or tasks, will be set to a higher value than the maximum value of $a_{i j}$ between all the pairs of real candidate trucks and tasks. Without the loss of generality, assume that the number of candidate tasks is larger than or equal to that of trucks $(m \geq n)$. Then, for given sets of candidate tasks and trucks, the minimum cost dispatching problem, which is an assignment problem, can be formulated as follows:

(P1)

$$
\begin{aligned}
& \text { Minimize } \sum_{\mathrm{i}=1}^{m} \sum_{\mathrm{j}=1}^{m} \mathrm{a}_{\mathrm{ij}} \mathrm{x}_{\mathrm{ij}} \\
& \text { subject to } \\
& \sum_{\mathrm{j}=1}^{\mathrm{m}} \mathrm{x}_{\mathrm{ij}}=1 \text {, for all } \mathrm{i}, \\
& \sum_{\mathrm{i}=1}^{\mathrm{m}} \mathrm{x}_{\mathrm{ij}}=1 \text {, for all } \mathrm{j}, \\
& \mathrm{x}_{\mathrm{ij}} \geq 0 \text {, for all } \mathrm{i} \text { and } \mathrm{j} .
\end{aligned}
$$

The dual problem of the above formulation becomes

(D)

$$
\operatorname{Minimize} \sum_{i=1}^{m} v_{i}-\sum_{j=1}^{m} p_{j}
$$

subject to

$\mathrm{v}_{\mathrm{i}}-\mathrm{p}_{\mathrm{j}} \geq-\mathrm{a}_{\mathrm{ij}}$ for all $\mathrm{i}$ and $\mathrm{j}$.

Property 1. (The optimality of a solution.) If the following two conditions hold, then the assignment is optimal: (1) The assignment is feasible; (2) $\mathrm{v}_{\mathrm{i}}=\mathrm{p}_{\mathrm{j}}-\mathrm{a}_{\mathrm{ij}}=\max _{\mathrm{k}=1, \cdots, \mathrm{m}}$ $\left\{\mathrm{p}_{\mathrm{k}}-\mathrm{a}_{\mathrm{ik}}\right\}$ for all $\mathrm{i}$ and $\mathrm{j}$ such that $\mathrm{x}_{\mathrm{ij}}=1$.

Proof. The fact that $v_{i}=p_{j}-a_{i j}=\max _{k=1}, \cdots, m\left\{p_{k}-a_{i k}\right\}$ for all $\mathrm{i}$ implies that $\mathrm{v}_{\mathrm{i}} \geq \mathrm{p}_{\mathrm{k}}-\mathrm{a}_{\mathrm{ik}}$ for all $\mathrm{i}$ and $\mathrm{k}$, which means that the set of $v_{i}$ and $p_{k}$ is feasible for constraint (1). Also, the fact that $\mathrm{v}_{\mathrm{i}}=\mathrm{p}_{\mathrm{j}}-\mathrm{a}_{\mathrm{ij}}$ for all $\mathrm{i}$ and $\mathrm{j}$ with positive $\mathrm{x}_{\mathrm{ij}}$ implies that the complementary slackness conditions, 
$\left(\mathrm{v}_{\mathrm{i}}-\mathrm{p}_{\mathrm{j}}+\mathrm{a}_{\mathrm{ij}}\right) \mathrm{x}_{\mathrm{ij}}=0$ for all $\mathrm{i}$ and $\mathrm{j}$, are satisfied. Thus, the conclusion holds. Q.E.D.

It will be assumed that the condition $\mathrm{v}_{\mathrm{i}}=\mathrm{p}_{\mathrm{j}}-\mathrm{a}_{\mathrm{ij}}=$ $\max _{\mathrm{k}}=1, \cdots, \mathrm{m}\left\{\mathrm{p}_{\mathrm{k}}-\mathrm{a}_{\mathrm{ik}}\right\}$ is satisfied if $\mathrm{v}_{\mathrm{i}}=\mathrm{p}_{\mathrm{j}}-\mathrm{a}_{\mathrm{ij}} \geq \max _{\mathrm{k}=1, \cdots, \mathrm{m}}$ $\left\{\mathrm{p}_{\mathrm{k}}-\mathrm{a}_{\mathrm{ik}}\right\}-\varepsilon$ for a very small positive real number $\varepsilon$. This modification is made to devise a solution procedure that guarantees termination within a finite number of iterations. Note that the effects of this modification on the objective value will be infinitesimal.

The price of each delivery-which a shipper pays to a truck driver-is determined through an auction process. During the auction process, each truck driver selects the delivery task that maximizes its own margin which is the price of the task minus the cost of performing the task, while each shipper chooses the truck that requests the least compensation which is the transportation cost required by the truck for performing the task plus the minimum margin requested by the truck. When trucks are assigned and prices and margins are determined optimally, a truck cannot increase its margin by changing its currently assigned task and a delivery task cannot decrease the compensation by changing its currently assigned truck.

A dispatching decision process is initiated whenever a truck is loaded or whenever a new delivery task arrives at the system. When a truck is loaded, the ASSIGN-TASK-TO-A-NEW-TRUCK procedure is initiated to secure the next delivery task. In case a delivery task is posted, the ASSIGN-TRUCK-TO-A-NEW-TASK procedure is initiated. Once either of the two procedures is initiated, the optimal prices and assignments are obtained.

During the ASSIGN-TASK-TO-A-NEW-TRUCK procedure, prices of delivery tasks decrease, while margins of trucks also decrease. However, during the ASSIGN-TRUCK-TO-A-NEW-TASK procedure, prices of delivery tasks increase, while margins of trucks also increase. The price of each delivery task is limited by an upper bound on the price pre-specified by the corresponding shipper, while the lower bound on the margin of a truck is also specified by the driver of the truck.

The following introduces two procedures (ASSIGNTASK-TO-A-NEW-TRUCK, ASSIGN-TRUCK-TO-ANEW-TASK) for optimally matching trucks with delivery tasks. Note that the number of available trucks (n) may not be the same as the number of available delivery tasks (m). This paper assumes that some unattractive delivery tasks may not be served. When a task is not assigned to a truck for a pre-specified time period, the task may be removed from the set of candidate tasks. When the number of trucks is larger than the number of delivery tasks, in which case some trucks may remain idle until a new task arrives at the system.

From the assumption that $m \geq n$, even after an assignment is determined, one or more tasks may not be assigned ("unassigned and inactive": UI) to a truck. Both the number of trucks and the number of tasks in the "assigned (A)" state are $n$. However, when a truck (task) becomes a newly candidate for an assignment, it is "unassigned" but has a potential to be assigned. The truck (task) is said to be "unassigned but activated (UA)." Also, during the assignment procedure, a less competitive truck (task) may have its assigned task (truck) taken away by another more competitive truck (task). Then, the former truck (task) becomes "unassigned but activated (UA)," while the latter truck (task) becomes "assigned (A)."

The auction process (ASSIGN-TASK-TO-A-NEWTRUCK) for the case of the truck initiation (when a new truck becomes idle) is illustrated in Figure 2. Before the new truck becomes a candidate of dispatching, three trucks are assigned to three tasks. Thus, they are in state of " $\mathrm{A}$ " except one task that is not assigned to any truck and so is in state "UI" (see Figure 2(a)). When a truck (truck 4) becomes a candidate, truck 4's initial state is "UA," and it seeks for a delivery task to perform (see Figure 2(b)). As illustrated in Figure 2, among waiting tasks, truck 4 selects a task (task c) giving the maximum margin at the current price. Then, truck 4 submits a bid with a price lower than the current price of task $\mathrm{c}$. Then, task $\mathrm{c}$ accepts the bid because the suggested price is lower than the current price, and informs the cancellation of assignment to the truck (truck 4) that task c was previously assigned to. The state of truck 4 is changed from "UA" to " $A$ " and the state of truck 3 is changed from "A" to "UA" (see Figure 2(c)). Then, truck 3 whose state became "UA" searches for the task that gives the highest margin at the current price. In Figure 2, it is task d. Because task d was in state of "UI," no truck turns to "UA" and so the auction process is terminated (see Figure 2(d)).

The following describes how to determine bidding prices in more detail. First, the procedure of ASSIGNTASK-TO-A-NEW-TRUCK is described.

Let A(i) be the set of tasks that can be assigned to truck i. And let the initial price of task $\mathrm{j}$ be $\mathrm{p}_{\mathrm{j}}^{0}$ for all $\mathrm{j}$. The initial state of the newly available truck is set to $U A$.

\section{(Procedure for a newly available truck: ASSIGN- TASK-TO-A-NEW-TRUCK)}

The following procedure is repeated until no $U A$ truck is found:

\section{(Preparing a bid by a $U A$ truck)}

Let the $U A$ truck be truck i. Compute the current margin (profit) that truck $i$ can earn by performing task $j \in A(i)$, which is given by

$$
\mathrm{v}_{\mathrm{ij}}=\max \left\{\mathrm{p}_{\mathrm{j}}-\mathrm{a}_{\mathrm{ij}}, 0\right\} .
$$

Find the best task $\mathrm{j}^{*}$ having the maximum value of $\mathrm{v}_{\mathrm{ij}}{ }^{*}=$

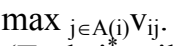

(Task $\mathrm{j}^{*}$ will give truck $i$ the maximum margin if it is assigned to truck i.)

If $v_{\mathrm{ij}^{*}}=0$, then this implies that there is no profitable task 
for truck i (This happens when prices of tasks in A(i) are too low for truck i to obtain a positive margin by performing any task in A(i). In this case, truck i should remain unassigned).

Let $\mathrm{v}_{\mathrm{i}}=0$ and the state of truck $\mathrm{i}$ be $U I$, then stop.

Otherwise, find the highest margin offered by tasks other than $j^{*}$, which is given by

$$
\mathrm{W}_{\mathrm{ij} *}=\max _{\mathrm{j} \in \mathrm{A}(\mathrm{i}), j \neq j * \mathrm{j}^{*} \mathrm{ij} \cdot}
$$

If task $\mathrm{j}^{*}$ is the only task in $\mathrm{A}(\mathrm{i})$, then set $\mathrm{w}_{\mathrm{ij} \text { * }}$ set to be 0 $\left(\mathrm{w}_{\mathrm{ij} *}{ }^{*}\right.$ means the margin that truck $\mathrm{i}$ can earn when it is assigned to the second best task).

Let the state of truck i be $A$. Compute the bid of truck i for task $j^{*}$, which is given by

$$
\mathrm{b}_{\mathrm{ij}}=\mathrm{p}_{\mathrm{j}^{*}-\mathrm{V}_{\mathrm{ij}} *}+\mathrm{W}_{\mathrm{ij}} *-\varepsilon .
$$

$\left(b_{i j *}\right.$ is the level of the price of task $j^{*}$ that gives the same margin to truck $i$ as the second best task of (3) does.)

Submit the bid to the manager of task $j^{*}$.

(Accepting the bid submitted by the manager of task $j$ *) If there is a truck that is currently assigned to task $j^{*}$ (let it be truck $\left.i^{*}\right)$, then make the state of truck $i^{*}$ be $U A$. Assign task $\mathrm{j}^{*}$ to truck $\mathrm{i}$ and change the state of truck $\mathrm{i}$ to $A$.

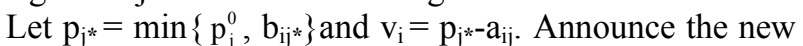
assignment and the revised data to all the tasks and trucks.

The following describes ASSIGN-TRUCK-TO-ANEW-TASK, which is the procedure triggered when a new delivery task becomes available:

\section{(Procedure for unassigned tasks: ASSIGN-TRUCK-} TO-A-NEW-TASK)

Let $\mathrm{V}(\mathrm{k})$ be the set of trucks that can be assigned to delivery task $\mathrm{k}$.

The following procedure is repeated until no $U A$ task is found:

\section{(Preparing a bid by a $U A$ task)}

Let the $U A$ task be task $\mathrm{k}$.

Compute the current minimum compensation required to induce each truck $i \in V(k)$, which is given by

$$
\mathrm{c}_{\mathrm{ik}}=\min \left\{\mathrm{v}_{\mathrm{i}}+\mathrm{a}_{\mathrm{ik}}, \mathrm{p}_{\mathrm{k}}^{0}\right\} .
$$

(Note that $\mathrm{v}_{\mathrm{i}}$ represents the margin that truck i already secured.)

Find the best truck $\mathrm{i}^{*}$ having the minimum value

$$
\mathrm{c}_{\mathrm{i}{ }^{*} \mathrm{k}}=\min _{\mathrm{i} \in \mathrm{V}(\mathrm{k})} \mathrm{c}_{\mathrm{ik}} \text {. }
$$

If $\mathrm{c}_{\mathrm{i}^{*} \mathrm{k}}=\mathrm{p}_{\mathrm{k}}^{0}$, then this implies that there is no affordable truck, in which case let $\mathrm{p}_{\mathrm{k}}=\mathrm{p}_{\mathrm{k}}^{0}$ and the state of task k be $U I$, then stop. (Task $\mathrm{k}$ will remain unassigned.)

Otherwise, find the minimum compensation for trucks other than truck $\mathrm{i}^{*}$

$$
\mathrm{d}_{\mathrm{i} * \mathrm{k}}=\min _{\mathrm{i} \in \mathrm{V}(\mathrm{k}), \mathrm{i} \neq \mathrm{i}^{*} \mathrm{c}_{\mathrm{ik}}} .
$$

$\left(\mathrm{d}_{\mathrm{i}^{*} \mathrm{k}}\right.$ means the compensation that task $\mathrm{k}$ must pay so that it is to be assigned to the second best truck.) If truck $i^{*}$ is the only truck in $\mathrm{V}(\mathrm{k})$, let $\mathrm{d}_{\mathrm{i}^{*} \mathrm{k}}=\mathrm{p}_{\mathrm{k}}^{0}$. Let the state of task k be $A$ Compute the bid of task $\mathrm{k}$ for truck $\mathrm{i}^{*}$, which is given by

$$
\mathrm{e}_{\mathrm{i}^{*} \mathrm{k}}=\mathrm{v}_{\mathrm{i}^{*}-\mathrm{c}{ }^{*} \mathrm{k}}+\mathrm{d}_{\mathrm{i}^{*} \mathrm{k}}+\varepsilon
$$

$\left(\mathrm{e}_{\mathrm{i}^{*} \mathrm{k}}\right.$ is the level of the margin that truck $\mathrm{i}^{*}$ can earn when task $\mathrm{k}$ pays the same compensation to truck $i^{*}$ as task $\mathrm{k}$ needs to do for inducing the second best truck of (7))

Submit the bid to the manager of truck $i^{*}$.

(Accepting the bid submitted by the driver of truck i*) If there is a task that is currently assigned to truck $i^{*}$ (let it be task $\mathrm{j}^{*}$ ), change the state of task $\mathrm{j}^{*}$ to $U A$. Assign truck $\mathrm{i}^{*}$ to task $\mathrm{k}$. Let $\mathrm{v}_{\mathrm{i}^{*}}=\min \left\{\mathrm{e}_{\mathrm{i}^{*} \mathrm{k}}, \mathrm{p}_{\mathrm{k}}^{0}-\mathrm{a}_{\mathrm{i}^{*} \mathrm{k}}\right\}$ and $\mathrm{p}_{\mathrm{k}}=$ $\mathrm{v}_{\mathrm{i}^{*}}+\mathrm{a}_{\mathrm{i}^{*} \mathrm{k}}$.

Announce the new assignment and the revised data to all the tasks and trucks.

Property 2: Suppose $p_{k}^{0}>a_{i k}$ for all $i$ and $k$. Then, procedures of ASSIGN-TASK-TO-A-NEW-TRUCK and ASSIGN-TRUCK-TO-A-NEW-TASK provide the optimal assignment to (P1) for a given set of available trucks and tasks in a finite number of iterations.

Proof: See Appendix.

The auction process solves the assignment problem optimally for a given set of delivery tasks and trucks. However, when a new truck or a new delivery task becomes a candidate for the assignment, the optimality-in property 2-of the solution from the previous auction process is no longer valid. Thus, the arrival event of a new candidate truck or a new delivery task initiates a new round of the auction process so that the optimality of the solution can be recovered. This procedure is similar to the method of the rolling planning horizon in the production planning.

Also, during the auction process, factors such as intentionally distorted bids and delayed responses by truck managers or task managers may cause the solution to deviate from the optimality condition. However, the economic motives of associated agents will keep these disturbances to a minimum. 


\section{A SIMULATION EXPERIMENT}

A simulation experiment was performed for a transportation system in which 100 independent trucks perform deliveries and tasks arrive dynamically. Each delivery task has a time window for delivery. Trucks become involved in the dispatching process for the next delivery task when they arrive at the pickup location of the current task. They are involved in the dispatching process until they are assigned a delivery task. Delivery tasks become involved in the dispatching process from the moment that they arrive at the brokerage system. The tasks remain involved in the dispatching process until the deadlines for the deliveries expire.

The dispatching decision process is triggered by the arrival of a new delivery task or the arrival of a truck at the pickup point of a previously assigned task. When a new delivery task arrives at the system, the ASSIGNTRUCK-TO-A-NEW-TASK process is initiated. When a truck arrives at the pickup position of a previously assigned task, the ASSIGN-TASK-TO-A-NEW-TRUCK process is triggered. Obviously, the dispatching decision may change whenever a new task arrives at the system or a new truck becomes a candidate for the dispatching. However, the decision about the next task for a specific truck is fixed when the truck completes the previous delivery task. When a truck completes a delivery task and finds no assigned task available, then it remains idle at the location where it completed the last task.

The delivery tasks are assumed to be randomly generated on a square of $10,000 \times 10,000$ (distance unit) ${ }^{2}$. For a delivery task, pickup and delivery positions are generated randomly on the square. The time window, [a, b], during which a delivery is allowed, was also generated randomly so that $a$ follows UNIFORM (CT + PT, $\mathrm{CT}+\mathrm{PT}+1)$ and $b$ follows UNIFORM $(\mathrm{CT}+\mathrm{PT}+50, \mathrm{CT}+$ $\mathrm{PT}+100$ ), where $\mathrm{CT}$ is the time when the task was generated and PT is the time required for a truck to travel from its pickup location to its delivery location. Tasks that were not completed within their time windows were removed from the system. The inter-arrival time of delivery tasks was assumed to follow the exponential distribution. The travel speed of trucks was assumed to be 50 distance units per unit time. The Euclidean distance travel was assumed for trucks. The time for loading and unloading was ignored because it has no effect on the dispatching decision. The initial prices for all tasks were set to 5,000 .

The simulation study was conducted for various average inter-arrival times of delivery tasks. The average inter-arrival time ranged between 1 and 2 time units. For each simulation condition, the simulation was run 10 times. The simulation time was set to 5,000 time units per run, and first 1,000 time units were considered to be the warm-up period. The simulation model was developed by using ARENA 3.5, which is a commercial simulation package.

As a reference for comparison, a simulation study using the "shortest distance rule" (SDR), which is the most popular rule in practice, was also performed. When the SDR is used, whenever a truck becomes idle or a new task is created, the rule searches for unassigned tasks or trucks that satisfy various constraints. If no unassigned task or truck exists, the truck or the new task waits. Otherwise, it selects the task or the truck that is located nearest to its position. Thus, the dispatching decision is always made in a one-to-one or one-to-many manner. The results are shown in Table 1.

In applying the distributed dispatching method, two different objectives were pursued. In the first experiment, the total empty travel time (ETT) was the objective to be minimized (MIN-ETT method). The empty travel time, which is the travel time from the delivery location of the current task that truck $i$ is performing to the pickup location of task $j$, was used as $a_{i j}$ shown in section 2. In the second experiment, the objective was the total response time (RT) for all delivery tasks. The response time is the time needed by a truck to travel from its current position to the pickup location of a delivery task. This means that in the MIN-RT method, the response time needed by truck $i$ for delivery task $j$ was used as $\mathrm{a}_{\mathrm{ij}}$ shown in section 3. The MIN-ETT method attempts to minimize the empty travel distance (time), while the MIN-RT method attempts to minimize the response time of delivery tasks. The MIN-ETT is expected to minimize the total cost from the viewpoint of trucks (see Table 2), while the MIN-RT is expected to maximize the throughput rate of the entire transportation system (see Table 3).

The following presents a comparison of various

Table 1. The simulation results of the SDR method

\begin{tabular}{lrrrrr}
\hline \multicolumn{1}{c}{ Average inter-arrival time } & 1 & 1.25 & 1.5 & \multicolumn{1}{c}{1.75} & \multicolumn{1}{c}{2} \\
\hline Total number of tasks generated & 3,973 & 3,151 & 2,641 & 2,266 & 1,983 \\
\hline Ratio of delivered tasks to all generated tasks & 76.9 & 87.3 & 95.0 & 100.0 & 100.0 \\
\hline Average response time per task & 42 & 51 & 57 & 30 & 21 \\
\hline Average empty travel time per task & 26 & 40 & 50 & 30 & 21 \\
\hline Average number of trucks involved in the dispatching process & 0.01 & 0.37 & 2.75 & 23.54 & 37.09 \\
\hline Average number of tasks involved in the dispatching process & 21.1 & 12.2 & 6.0 & 0.9 & 0.8 \\
\hline
\end{tabular}


Table 2. The simulation results of the MIN-ETT method

\begin{tabular}{lrrrrr}
\hline \multicolumn{1}{c}{ Average inter-arrival time } & 1 & 1.25 & 1.5 & 1.75 & 2 \\
\hline Total number of tasks generated & 3,973 & 3,151 & 2,641 & 2,266 & 1,983 \\
\hline Ratio of delivered tasks to all generated tasks & 80.2 & 93.2 & 97.4 & 99.0 & 98.9 \\
\hline Average response time per task & 53 & 46 & 39 & 34 & 31 \\
\hline Average empty travel time per task & 19 & 20 & 18 & 17 & 16 \\
\hline Average number of trucks participating in the dispatching process & 84.1 & 85.2 & 88.0 & 90.2 & 91.5 \\
\hline Average number of tasks involved in the dispatching process & 41.0 & 23.5 & 15.0 & 10.7 & 8.3 \\
\hline Average committed price of tasks & 3,396 & 2,184 & 1,630 & 1,384 & 1,306 \\
\hline Average margin realized for trucks & 2,421 & 1,207 & 734 & 549 & 491 \\
\hline Average cost for delivery tasks & 975 & 977 & 896 & 836 & 815 \\
\hline Average number of iterations performed per a round of dispatching & 7.9 & 2.3 & 1.5 & 1.3 & 1.2 \\
\hline
\end{tabular}

Table 3. The simulation results of the MIN-RT method

\begin{tabular}{lrrrrr}
\hline \multicolumn{1}{c}{ Average inter-arrival time } & 1 & 1.25 & 1.5 & 1.75 & 2 \\
\hline Total number of tasks generated & 3,973 & 3,151 & 2,641 & 2,266 & 1,983 \\
\hline Ratio of delivered tasks to all generated tasks & 81.1 & 94.7 & 98.5 & 99.5 & 99.4 \\
\hline Average response time per task & 50 & 38 & 28 & 23 & 21 \\
\hline Average empty travel time per task & 20 & 23 & 21 & 20 & 19 \\
\hline Average number of trucks involved in the dispatching process & 83.7 & 82.6 & 85.6 & 88.5 & 90.4 \\
\hline Average number of tasks involved in the dispatching process & 37.7 & 14.8 & 5.7 & 2.8 & 2.0 \\
\hline Average committed price for a task & 3,414 & 2,345 & 1,776 & 1,516 & 1,410 \\
\hline Average margin realized by a truck & 2,367 & 1,163 & 671 & 514 & 463 \\
\hline Average cost per delivered task & 1,047 & 1,180 & 1,105 & 1,002 & 947 \\
\hline Average number of iterations performed per a round of dispatching & 5.0 & 2.1 & 1.2 & 1.1 & 1.0 \\
\hline
\end{tabular}

performance measures of three different dispatching methods.

\section{Throughput rate}

Other things being equal, because the higher throughput rate implies that more delivery tasks are satisfied, the rate must be an important measure of performance. Note that not all generated tasks can be delivered by trucks because of limited time windows and a limited number of trucks. Figure 3 shows how the ratio of the number of delivered tasks to the total number of generated tasks changes as the inter-arrival time of tasks changes. For all three cases, as expected, the ratio converged to $100 \%$ as the average inter-arrival time increased. In a lower range of the inter-arrival time, the MIN-ETT and the MIN-RT outperformed SDR significantly, although the SDR showed a slightly better performance when the inter-arrival time was longer than 1.75. Also, the difference between the SDR and the distributed dispatching method became the largest when the inter-arrival time was near 1.25 . When the interarrival time was too high or too low, it was more probable that the dispatching decision was made on a one-to- one or one-to-many basis. In such a case, the advantage of the look-ahead property of the distributed dispatching seemed to diminish.

\section{The average empty travel time}

In trucking systems, the empty travel time is an important performance measure, because the travel time significantly affects the operating cost. Figure 4 compares the average empty travel time for the three dispatching methods. Note that the MIN-ETT and the MINRT significantly outperformed the SDR over the entire range of the average inter-arrival time. The difference was the largest when the average inter-arrival time was about 1.5 . When the average inter-arrival time was too short or too long, there was a high possibility that the matching was done for one truck with one of many waiting tasks or for one task with one of many waiting trucks. In these two extreme cases, the dispatching decision generated by the distributed methods would not be much different from that found by the SDR. Comparing the two distributed dispatching methods with each other, the MIN-ETT showed a slightly less empty travel time than did the MIN-RT. 


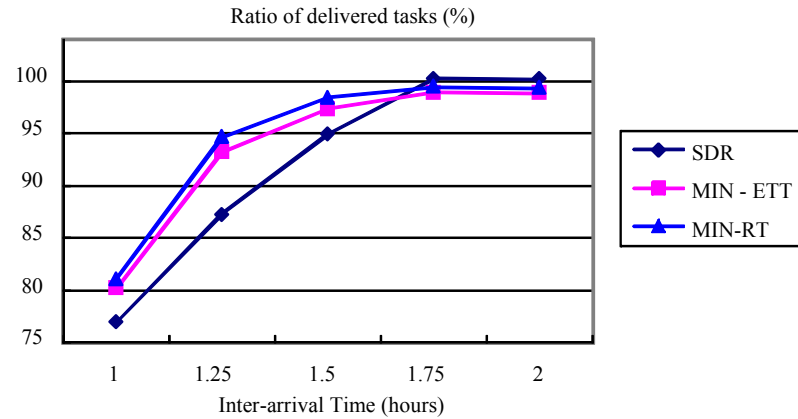

Figure 3. The ratio of delivered tasks under various dispatching rules.

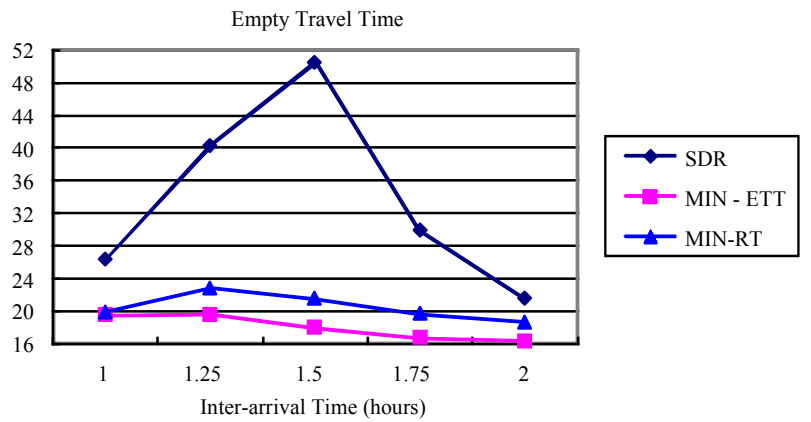

Figure 4. The average empty travel time for various dispatching rules.

\section{Response time}

The response time of a task is the time between the moment when a vehicle is assigned a task and the moment when the vehicle arrives at the pickup location of the task. Figure 5 shows the results of the simulation experiment. In case of the SDR, the average response time was the highest when the average inter-arrival time was near 1.5. Note that the average empty travel time was also the highest when the average inter-arrival time was near 1.5. It was shown that the MIN-RT outperforms the MIN-ETT in average response time, and the difference between the two methods increases as the average inter-arrival time increases. Figure 6 shows that as the arrival rate of tasks increases, the number of rejected tasks as well as the number of served tasks increases.

In trucking systems that consist of independent profit-makers, the committed price, which is obtained during the dispatching decision, can be used as a real monetary value that a shipper must pay to a trucker. In such a case, it is possible to calculate the margin for each delivery task that a truck driver can earn from performing the task. The margin will be the price of each task deducted by the cost. In this study, the cost related to the loaded travel was excluded from both the price and the cost. However, there will be no difference in the algorithms and the results of the experiment, even if the loaded travel cost is included in the cost model, because the loaded cost does not influence the dispatching decision.

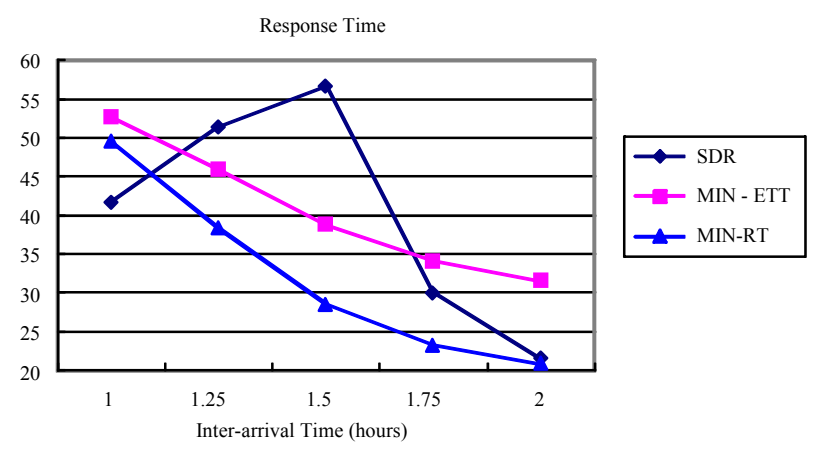

Figure 5. The average response time for various dispatching rules.

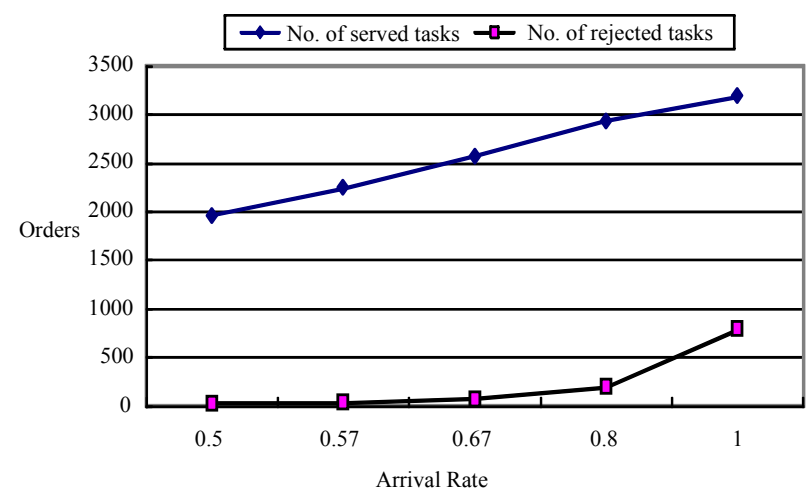

Figure 6. Changes in the number of rejected tasks with respect to the arrival rate of tasks in case of the MIN-ETT.

Figure 7 shows that the average final price, which is the price of a task when a truck is finally committed to the task, tends to increases as the arrival rate increases. As the average final price increases, the average margin per task also increases. The trends coincide with our intuition that the more intense competition among trucks for tasks will reduce the average profit margin. The margin is calculated by subtracting the cost from the revenue. Thus, the margin is negatively correlated to the cost. The average cost also increases as the arrival rate of tasks increases. This trend is due to the fact that, as the arrival rate increases, the number of candidate trucks per candidate delivery task decreases, and thus, the average empty travel distance increases.

When the auction price plays the role of a real transaction price, the next natural question may be how shippers can determine the maximum price of delivery tasks. Figure 8 shows that as the maximum price increases, the average final price and the average margin also increase. Note that within one simulation run, all the tasks are given the same maximum price. However, this does not mean that a lower maximum price necessarily results in more benefit for shippers. Figure 9 shows that the rejection ratio increases as the maximum price decreases. This indicates that although a lower maximum price will decrease the final price, it also increases the risk of rejection of the delivery task by trucks. 


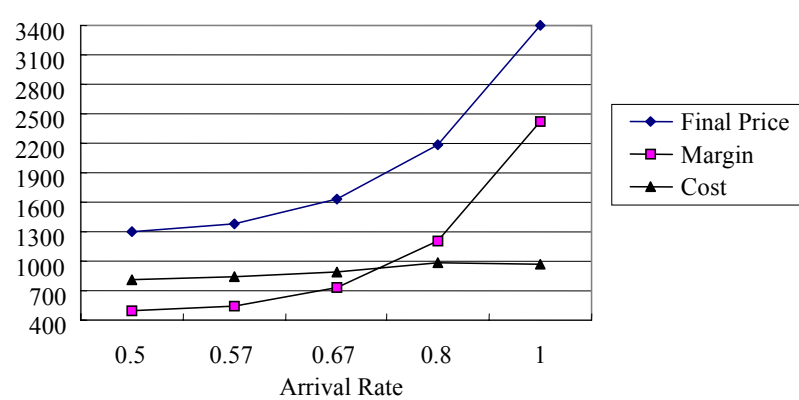

Figure 7. Changes in the final price, margin, and cost with respect to the arrival rate of tasks in case of the MIN-ETT.

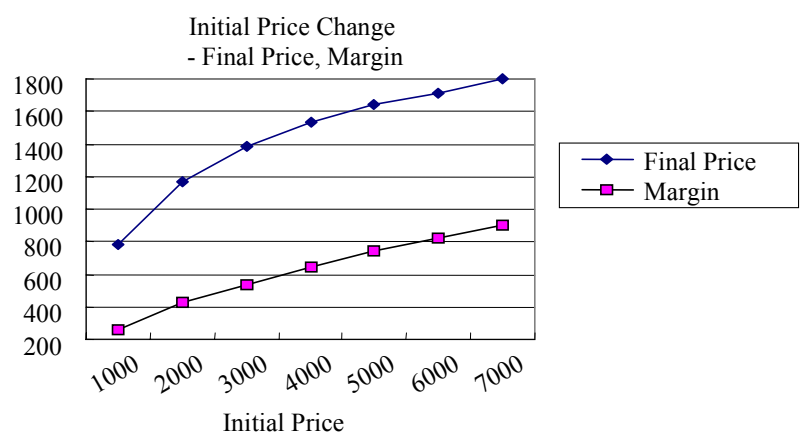

Figure 8. Changes in the average final price and the average margin with respect to the maximum price in case of the MIN-ETT.

Note also that the average number of iterationswhich is the number of bid constructions by truck drivers and shippers-is relatively very small comparing with the average number of trucks or tasks involved in the dispatching process. This implies that the effect of a new arrival of candidate truck or delivery task is confined to a small group of tasks and trucks.

In summary, as shown in Figure 10, benefits of shippers and truck drivers resulting from the participation into the brokerage system are made possible by the cost reduction from the optimized dispatching. Through the auction process, the final prices of transportation

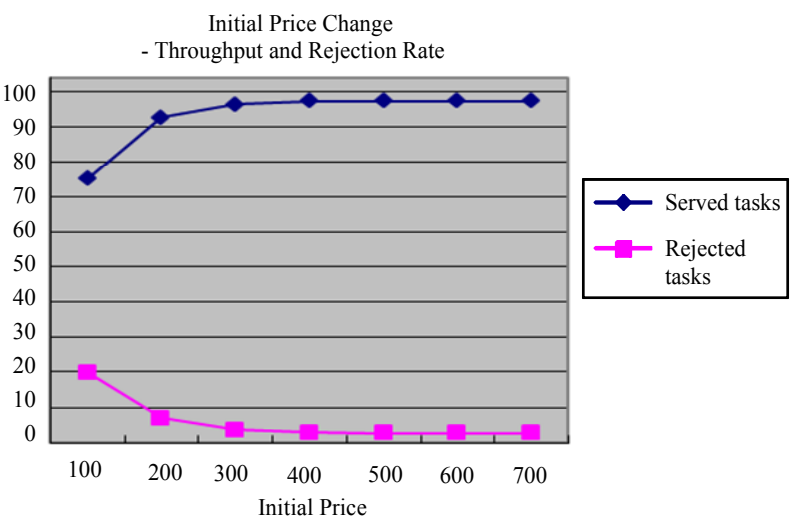

Figure 9. Changes in the ratio of served tasks and rejected tasks with respect to the maximum price in case of the MIN-ETT.

become lower than the initial maximum prices that shippers originally suggested. As a result, more shippers become to utilize the brokerage system, which means a higher utilization of participating trucks. The lowered price for delivery tasks may impair the profitability of trucks. However, the cost saving resulting from the reduction in the total empty travel distance by the optimized dispatching and the revenue increase from the higher utilization will outweigh the reduction in the revenue from the lowered prices.

\section{CONCLUSIONS}

A distributed dispatching method is suggested for large-scale pickup and delivery systems. A basic rationale for the distributed dispatching is provided. A truckinitiated dispatching procedure and a task-initiated dispatching procedure are suggested.

A simulation study was performed to test the performance of the suggested dispatching procedure. Two different versions of the distributed dispatching method were tested: One is the MIN-ETT method in which the total empty travel time is minimized; in the other dis-

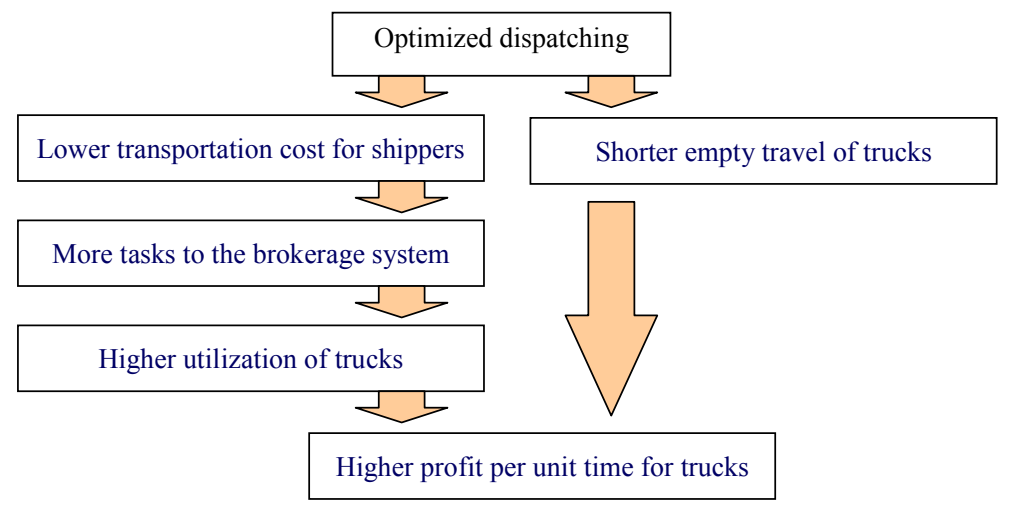

Figure 10. Incentive compatibility of the brokerage system. 
tributed dispatching procedure (MIN-RT), the total response time is minimized. The performances by the shortest distance rule (SDR) were compared with those by distributed dispatching procedures. The experiment results showed that the MIN-ETT and the MIN-RT significantly outperformed the SDR within most of the range of the average inter-arrival time.

For the case of the MIN-ETT, the experiment also analyzed how the arrival rate or the maximum prices of tasks influence the respective averages of the final price and the margin. The average final price and the average margin tend to decrease as the arrival rate of tasks decreases. It was also found that as the maximum price of tasks decreases, the final price and the margin also decrease, while the risk of rejection of the tasks increases.

It is expected that the pricing strategy of shippers and the cost structure will significantly influence the performance of the distributed algorithms and the economic interests among shippers and truckers, all about which further research is necessary.

\section{ACKNOWLEDGEMENT}

This work was supported for two years by Pusan National University Research Grant.

\section{REFERENCES}

Beilock, R., Shell, T. (1992) Brokerage and the potential for electronic marketing of produce transportation, Transportation Journal, 60-71.

Bertsekas, D. P. (1981), A new algorithm for the assignment problem, Mathematical Programming, 21, 152-171.

Bertsekas, D. P. (1988), The auction algorithm: a distributed relaxation method for the assignment problem, Annals of Operations Research, 14, 105-123.

Bertsekas, D. P. (1990), The auction algorithm for assignment and other network flow problems: a tutorial, Interfaces, 20(4), 133-149.

Carvalho, T. A. and Powell, W. B. (2000), A multiplier adjustment method for dynamic resource allocation problems, Transportation Science, 34(2), 150-164.

Fischer, K., Müller, J. P., and Pischel, M. (1996), Cooperative transportation scheduling: an application domain for DAI, Journal of Applied Artificial Intelligence, Special Issue on Intelligence Agents, 10(1).

Frantzeskakis, L. F. and Powell, W. B. (1990), A successive linear approximation procedure for stochastic, dynamic vehicle allocation problems, Transportation Science, 24(1), 40-57.

Gendreau, M., Guertin, F., Potvin, J.-Y., and Seguin, R. (2006), Neighborhood search heuristics for a dynamic dispatching problem with pick-ups and deliver- ies, Transportation Research Part C, 14, 157-174.

Gendreau, M., Laporte, G., and Séguin, R. (1995), An exact algorithm for the vehicle-routing problem with stochastic demand and customers, Transportation Science, 29(2), 143-154.

Gendreau, M., Laporte, G., and Séguin, R. (1996a), Stochastic vehicle routing, European Journal of Operational Research, 88, 3-12.

Gendreau, M., Laporte, G., and Séguin, R. (1996b), A tabu search heuristic for the vehicle routing problem with stochastic demands and customers, $O p$ erations Research, 44(3), 469-477.

Gribkovskaia, I., Halskau, Ø., Laporte, G., and Vl`cek, M. (2007), General solutions to the single vehicle routing problem with pickups and deliveries, European Journal of Operational Research, 180, 568584.

Gronalt, M., Hartl, R. F, and Reimann, M. (2003), New savings based algorithms for time constrained pickup and delivery of full truckloads, European Journal of Operational Research, 151(3), 520-535.

Ha, H. G. and Kim, Y. J. (2003), A Strategy for Promoting On-line Brokering Service in Field of Logistics, The Korea Transport Institute.

Ichoua, S., Gendreau, M., and Potvin, J.-Y. (2000), Diversion issues in real-time vehicle dispatching, Transportation Science, 34(4), 426-438.

Imai, A., Nishimura, E., and Current, J. (2007), A Lagrangian relaxation-based heuristic for the vehicle routing with full container load, European Journal of Operational Research, 176(1), 87-105.

Kutanoglu, E. and Wu, S. D. (1999), On combinatorial auction and Lagrangean relaxation for distributed resource scheduling, IIE Transactions, 31, 813-826.

Laporte, G. and Louveaux, F. V. (1993), The integer Lshaped method for stochastic integer programs with complete recourse, Operations Research Letters, 13, 133-142.

Madsen, O. B. G., Ravn, H. F., and Rygaard, J. M. (1995), A heuristic algorithm for a dial-a-ride problem with time windows, multiple capacities, and multiple objectives, Annals of Operations Research, 60, 193-208.

Parragh, S. N., Doerner, K. F., and Hartl, R. F. (2008a), A survey on pickup and delivery problems. Part I: transportation between customers and depot, Journal für Betriebswirtschaft, 58(1), 21-51.

Parragh, S. N., Doerner, K. F., and Hartl, R. F. (2008b), A survey on pickup and delivery problems. Part II: transportation between pickup and delivery locations, Journal für Betriebswirtschaft, 58(2), 81-117.

Powell, W. B. (1996), A stochastic formulation of the dynamic assignment problem with an application to truckload motor carriers, Transportation Science, 30(3), 195-219. 
Powell, W. B. and Carvalho, T. A. (1995), Dynamic fleet management as a logistics queueing network, Annals of Operations Research, 61, 165-188.

Powell, W. B. and Gittoes, D. (1996), An approximate labeling algorithm for the dynamic assignment problem, in: L. Bianco, P. Toth, M. Bielli, Eds., Advanced Methods in Transportation Analysis (Springer, New York).

Psaraftis, H. N. (1988), Dynamic vehicle routing problems, in: B. L. Golden and A. A. Assad Eds., Vehicle Routing: Methods and Studies (Elsevier Science).

Psaraftis, H. N. (1995), Dynamic vehicle routing: status and prospective, Annals of Operations Research, 61, 143-164.

Shen, Y., Potvin, J.-Y., Rousseau, J.-M., and Roy, S. (1995), A computer assistant for vehicle dispatching with learning capabilities, Annals of Operations Research, 61, 189-211.

Wang, X. and Regan, A. C. (2002), Local truckload pickup and delivery with hard time windows constraints, Transportation Research Part B: Methodological, 36(2), 97-112.

\section{APPENDIX: PROOF OF PROPERTY 2}

At the moment that either of the two procedures is triggered, the state of the system must be among one of the following four different cases:

Case 1: A truck becomes a new candidate for dispatching and the number of candidate trucks is smaller than or equal to the number of candidate tasks.

Case 2: A truck becomes a new candidate for dispatching and the number of candidate trucks is larger than the number of candidate tasks.

Case 3: A task becomes available and the number of candidate trucks is smaller than or equal to the number of candidate tasks.

Case 4: A new task becomes available and the number of candidate trucks is larger than the number of candidate tasks.

The assertion will be proved for each of the above four different cases in the following.

Case 1: Because $p_{k}^{0}>\mathrm{a}_{\mathrm{ik}}$ for all $\mathrm{i}$ and $\mathrm{k}$, every truck can obtain a positive margin when it is assigned to task k. When a truck becomes newly available, there are (n-1) trucks of state " $A$, ," (n-1) tasks of state " $A$," and $(\mathrm{m}-\mathrm{n}+1)$ tasks of state " $U A$." The new truck arrives in " $U A$ " state and one truck remains in the state of " $U A$ " until a truck becomes assigned to one of $(m-n+1)$ tasks of state "UI." As soon as a truck is assigned to one of $(\mathrm{m}-\mathrm{n}+1)$ tasks of state "UI," the procedure is terminated. When the procedure is terminated, there exist $\mathrm{n}$ trucks of state " $A$, , $\mathrm{n}$ tasks of state " $A$," and m-n tasks of state "UI." At every iteration, based on equation (4), the price of one assigned task decreases by $\mathrm{v}_{\mathrm{ij}} *-\mathrm{W}_{\mathrm{ijj}} *+\varepsilon(>>\varepsilon>0)$. Thus, in the long run, a task of state "Ur" must be selected as the best task $\left(j^{*}\right)$ by a truck of state "UA." Thus, the procedure terminates in a finite number of iterations. Once a truck submits a bid to a task, it means that $\mathrm{v}_{\mathrm{i}}=\max _{\mathrm{k}=1, \ldots}$ $\mathrm{m}\left\{\mathrm{p}_{\mathrm{k}}-\mathrm{a}_{\mathrm{ik}}\right\}$, which is condition (2) of Property 1, and remains satisfying the condition until the truck becomes "UA." This is due to the fact that the price of no other task increases during the iteration. For the case of dummy trucks, the cost of assignment to every task is the same, and the prices of unassigned tasks are $p_{k}^{0}$, which is high enough to enable dummy trucks to have a positive margin. Thus, for any assignment of dummy trucks to tasks, condition (2) of Property1 is satisfied. Also, note that the assignment between trucks and tasks remains feasible during the entire process of the dispatching. Thus, the conclusion holds, based on Property1.

Case 2: When the number of available trucks is larger than the number of available tasks, (n-m) trucks become "unassigned" and cannot find a task that results in a positive margin. Because the prices of dummy tasks are the same and the cost of assignment of a truck to every dummy task is the same, for any assignment of unassigned trucks to dummy tasks, condition (1) of Property 1 is satisfied. Thus, the conclusion holds, based on Property1.

In the same way, the proof for cases 3 and 4 can be derived from the perspective of tasks instead of trucks and thus will be omitted here. 